\title{
Porque diez años no son nada: de @tic revista d'innovació educativa a Research in Education and Learning Innovation Archives
}

\author{
Because ten years is nothing: from @tic revista d'innovació educativa to Research in \\ Education and Learning Innovation Archives \\ Perquè deu anys no són res: de @tic revista d'innovacio educativa a Research in \\ Education and Learning Innovation Archives
}

\author{
Paz Villar-Hernández* \\ Servei de Formació Permanent i Innovació Educativa, Universitat de València, Valencia, España \\ *Autor para correspondencia: paz.villar@uv.es (Paz Villar-Hernández)
}

| Publicado: 24/06/2019

RESUMEN: Research in Education and Learning Innovation Archives (REALIA) es el nuevo nombre de la revista que desde 2008 y hasta 2018 publicó el Servei de Formació i Innovació Educativa de la Universitat de València.. Al igual que antes lo han hecho otras, en 2019 @tic revista d'innovació educativa ha cambiado su nombre. En este texto se repasan los diez años de historia de @tic revista d'innovació educativa, se exponen los motivos de ese cambio y se presentan las novedades introducidas y algunas perspectivas de futuro.

PALABRAS CLAVE: editorial; revistas científicas; @tic revista d'innovació educativa; Research in Education and Learning Innovation Archives

\section{Cómo citar:}

Villar-Hernández, P. (2019).

Porque diez años no son

nada: de @tic revista

d'innovació educativa a

Research in Education and

Learning Innovation

Archives. Research in

Education and Learning

Innovation Archives,

22,1-10.

10.7203/realia.22.15379

Copyright: El/La Autor/a. Open Access: Este es un artículo de acceso abierto distribuido bajo los términos de la licencia Creative Commons AttributionNonCommercial-ShareAlike 4.0 International (CC BY-NC-SA 4.0)

Financiación: None informed
ABSTRACT: Research in Education and Innovation Innovation Archives (REALIA) is the new name of the journal that from 2008 to 2018 published the Servei de Formació i Innovació Educativa of the Universitat de València. As others have done before, in 2019 @tic revista d'innovació educativa changed its name. In this text we review the ten years of @tic revista d'innovació educativa, we explain the reasons for this change and introduce new features and some future prospects.

KEYWORDS: editorial; scientific journals; @tic revista d'innovació educativa; Research in Education and Learning Innovation Archives

RESUM: Research in Education and Learning Innovation Archives (REALIA) és el nou nom de la revista que des de 2008 i fins a 2018 va publicar el Servei de Formació i Innovació Educativa de la Universitat de València. Igual que abans ho han fet unes altres, en 2019 @tic revista d'innovació educativa ha canviat el seu nom. En aquest text es repassen els deu anys d'història de @tic revista d'innovació educativa s'exposen els motius d'aquest canvi i es presenten les novetats introduïdes i algunes perspectives de futur.

PARAULES CLAU: editorial; publicacions científiques; @tic revista d'innovació educativa; Research in Education and Learning Innovation Archives 


\section{INTRODUCCIÓN}

Para conocer los orígenes de las primeras revistas educativas españolas debemos remontarnos a los primeros años del siglo pasado. Ubicadas en algunos casos entre la divulgación científico-pedagógica y la ciencia propiamente dicha, aparecieron a finales del s. XIX y primer cuarto del s. XX publicaciones esenciales para el conocimiento de las corrientes europeas de pensamiento pedagógico y la sistematización del pensamiento propio; entre ellas ocupan un lugar singular el Boletín de la Institución Libre de Enseñanza (1877) dirigido por Francisco Giner de los Ríos, la Revista de Pedagogía (1922) fundada por Lorenzo Luzuriaga, la Revista de Escuelas Normales (1923) que ese mismo año apuntaba a Modesto Bargallo como su Redactor Especial, o la Revista de Psicología i Pedagogía (1933-1937) fundada por Emilio Mira.

García-Hoz (1980) en un clarificador artículo sobre los dos tipos de pensamiento pedagógico que coexistieron en España durante el siglo XX (el que denomina "intuitivo" y al que se refiere como "científico") recorre el fecundo camino que hizo crecer la investigación propiamente científica en nuestro país. Y en este recorrido son mencionadas aquellas revistas que ayudaron a difundir la investigación científicopedagógica. García-Hoz (1980) coincide con Ruiz-Corbella, Galán, y Diestro (2014, p. 5) al señalar a la Revista Española de Pedagogía (1943) y a Bordón. Revista de Pedagogía (1949) como las revistas educativas científicas españolas por excelencia, si bien estos últimos añaden el nombre de la Revista Nacional de Educación (1941), transformada en Revista de Educación en 1952 y editada tradicionalmente por el ministerio responsable de esta área.

En el terreno digital fue la revista RELIEVE (Revista ELectrónica de Investigación y EValuación Educativa), editada por la Asociación Interuniversitaria de Investigación Pedagógica (AIDIPE) el primer actor educativo en abrir este camino al resto en los años 90. En concreto, el 14 de enero de 1995 esta publicación hacía llegar su primer número por correo electrónico a sus lectores (Aliaga, 2014, p. 5). AIDIPE ya contaba en esos momentos con otra publicación impresa de renombre: la Revista de Investigación Educativa, que publicaba desde 1983 textos de investigación educativa de perfil generalista. Y, por supuesto, y a pesar de las reticencias y críticas iniciales, la realidad es que la edición electrónica, el modelo de revista electrónica, se ha impuesto; de hecho lo que ahora se plantea es la desaparición del modelo de revista científica impresa (Delgado López-Cózar, 2015, p. 10).

Desde esa década el crecimiento en el número de revistas científicas españolas ha sido exponencial no solo en un sentido cuantitativo, sino también cualitativo (RuizCorbella et al., 2014).

Si en el año 2000 aún no había recogida ninguna revista de educación española en el índice de impacto bibliográfico por excelencia, el fournal Citation Report (RuizCorbella et al., 2014, p. 10), en el año 2010 estas suponían un 9,63\% (n=5), según los datos manejados por Moreno-Pulido, López-González, Rubio-Garay, Saúl, y SánchezElvira-Paniagua (2013). En 2018 de entre las 243 revistas que forman parte de la categoría "Education and Educational Research" del $\mathcal{F C R}$, tan solo 7 españolas fueron incluidas en alguno de sus cuartiles: Comunicar, Psicología Educativa, Educación XXI, Revista Española de Pedagogía, Enseñanza de las Ciencias, Porta Linguarum y Revista de Educación; estos datos quedan lejos de las 20 editadas en los Países Bajos e incluidas en él, pero es una progresión interesante y coherente con la profesionalización e internacionalización vivida por la edición científica española en las últimas décadas.

Si continuamos en el terreno cualitativo, vemos que el SCImago fournal Rank (SFR) incluyó 35 publicaciones españolas del área de Educación (selección: Social SciencesEducation-Western Europe-Journals) en su ranking de 2018. España fue de hecho en 2018 el tercer país en número de revistas de Educación dentro del $S 7 R$, número solo superado por las 286 de Reino Unido y las 40 de los Países Bajos, lugares por otra 
parte donde residen fiscalmente algunos de los más potentes grupos editoriales como Springer, Wiley, Taylor \& Francis, Elsevier, etc.

En cuanto a las causas que impulsaron la creación de las revistas españolas en esta área, Ruiz-Corbella et al. (2014) apuntan cuatro, y todas son consecuencia de los cambios normativos llevados a cabo por el Gobierno Español, que modificaron la estructura organizacional de las universidades y mostraron el camino hacia una valoración y evaluación del profesorado universitario basado en criterios de calidad investigadora.

En el caso de @tic revista d'innovació educativa dos aspectos impulsaron su creación: el primero tuvo que ver con las convocatorias de innovación educativa que la Universitat de València estaba financiando desde hacía años (la primera data del curso académico 2003-2004) (OCE-UV, s.f.) ; la segunda, con la vocación de difundir a través del acceso abierto contenidos de investigación educativa cuya forma inicial tenía mucho que ver con las experiencias en el aula de educación superior y con proyectos de desarrollo y aplicación de tecnologías educativas en el aula.

\section{HISTORIA DEL PASADO: @TIC REVISTA D'INNOVACIÓ EDUCATIVA EN PERSPECTIVA}

Así, en 2008 el Servei de Formació Permanent i Innovació Educativa (SFPIE) de la Universitat de València vinculado al Vicerrectorado de Convergencia Europea, creó la revista científica de acceso abierto @tic revista d'innovació educativa. Esta publicación se encuentra así dentro de ese $42 \%$ que Rodríguez-Yunta y Giménez-Toledo (2013, p. 37) apuntaban como editadas por universidades (y ese $38,1 \%$ de universidades públicas), en su estudio sobre revistas españolas de Humanidades y Ciencias Sociales.

Fue la primera revista específica de innovación educativa publicada por la Universitat de València, si bien sobre el área educativa muchas otras con vínculos editoriales próximos a la UV nos antecedieron (ver Tabla 1).

Tabla 1. Revistas del área educativa publicadas en el sistema Open Journal System de la Universitat de València

\begin{tabular}{|c|c|c|}
\hline Fundación & Título & Editor \\
\hline 1989 & Didáctica de las Ciencias Experimentales y Sociales & $\begin{array}{l}\text { Dpt. Didàctica de les Ciències Experimentals } \\
\text { i Socials }\end{array}$ \\
\hline 1995 & $\begin{array}{l}\text { RELIEVE - Revista Electrónica de Investigación y Evaluación } \\
\text { Educativa }\end{array}$ & AIDIPE \\
\hline 1998 & $\begin{array}{l}\text { LEEME. Revista Electrònica de la Llista Europea de Música a } \\
\text { I'Educació }\end{array}$ & Universitat de València \\
\hline 2003 & EARI Educación Artística Revista de Investigación & $\begin{array}{l}\text { Instituto Universitario de Creatividad e } \\
\text { Innovaciones Educativas de la Universitat de } \\
\text { València }\end{array}$ \\
\hline 2005 & Foro de profesores de $\mathrm{E} / \mathrm{LE}$ & $\begin{array}{l}\text { Foro de Profesores de E/LE de la Universitat } \\
\text { de València }\end{array}$ \\
\hline 2008 & Revista de Sociología de la Educación-RASE & $\begin{array}{l}\text { Comité de Investigación de Sociología de la } \\
\text { Educación de la Federación Española de } \\
\text { Sociología }\end{array}$ \\
\hline 2008 & $\begin{array}{l}\text { @tic revista d'innovació educativa (en 2019, Research in } \\
\text { Education and Learning Innovation Archives) }\end{array}$ & SFPIE. Universitat de València \\
\hline 2017 & Creativity and Educational Innovation Review (CEIR) & $\begin{array}{l}\text { Instituto Universitario de Creatividad e } \\
\text { Innovaciones Educativas de la Universidad } \\
\text { de Valencia }\end{array}$ \\
\hline 2018 & Journal of Literary Education & Universitat de València \\
\hline
\end{tabular}


Siguiendo a pioneras ya mencionadas como RELIEVE, @tic revista d'innovació educativa nació como revista exclusivamente electrónica en un momento en el que ya era evidente que este modelo había llegado para quedarse.

¿A qué hacía referencia ese título? En primer lugar, empleaba la arroba (@), símbolo asociado a la comunicación y a Internet por excelencia, e incorporaba las siglas de Tecnologías de la Información y la Comunicación (TIC) tan en boga en aquel momento y tan adecuada para una publicación que deseaba presentar propuestas donde estas tecnologías se trabajaran en el nivel educativo. Además, lo incorporaba a un concepto, que semánticamente hacía referencia a un espacio de observación en las alturas, el del ático (àtic en valenciano). Sin embargo en ese momento no se tuvieron en cuenta los enormes problemas que esa propuesta significaba para su internacionalización y su empleo en bases de datos.

@tic revista d'innovació educativa potenció inicialmente una investigación educativa basada en la experiencia docente. Desde un primer momento, el equipo editorial tuvo claro que dichos contenidos debían ser evaluados y juzgados por expertos nacionales e internacionales en las áreas objeto de análisis, de manera que tan solo aquellos que aportaban un valor añadido y contribuían al avance de la ciencia fueran objeto de difusión por parte de la revista.

Por otro lado en sus primeros años la revista quiso mantener cierta vocación de divulgación científica. Por ello, hasta la publicación del número 8 (Primavera, 2012) @tic revista d'innovació educativa aún incorporaba en su haber tres secciones no indexadas; eran las de: Evaluación de herramientas, Sala de lectura y La Pérgola.

La sección Evaluación de herramientas incorporaba manuscritos donde se presentaba el desarrollo de herramientas tecnológicas susceptibles de utilizar en la docencia. En esta sección aparecieron trabajos sobre Respondus, una comparativa de programas de creación de mapas conceptuales, se presentó una aplicación creada por la Consejería de Educación y Ciencia de Castilla La-Mancha denominada Cuadernia con la que se creaban cuadernos digitales, entre otras muchas otras tecnologías educativas.

La sección Sala de Lectura ofreció un espacio al análisis de libros e informes institucionales sobre las áreas que la revista trataba.

Pero sin duda la sección más novedosa fue la de La Pérgola, que presentaba trabajos de experiencias educativas de alumnado de grado y posgrado. Así, nuestros lectores pudieron conocer la experiencia educativa en una licenciatura de Química de tres alumnas de la Universitat de València o experiencias de alumnado Erasmus contadas por sus participantes.

Estas tres secciones desaparecieron en 2012, cuando el equipo editorial decidió redefinir el perfil de la revista, y ahondar en contenidos exclusivamente científicos.

En esta misma línea un tiempo antes al grupo inicial se incorporaron nuevos nombres de expertos nacionales e internacionales en ambos consejos: el Científico y el Editorial. Es esencial hacer una mención especial a todos ellos, porque sin su apoyo, orientación y ayuda la revista no habría podido proyectarse como lo ha hecho durante estos diez años. Otra mención especial va dirigida a esos académicos que han actuado como evaluadores de los artículos, tanto los publicados como los que se han quedado en el camino. Sin su ayuda desinteresada habría sido imposible publicar ningún número, pero no es tan solo eso. La realidad es que muchos hicieron contribuciones de gran calado y permitieron que esos manuscritos que nos llegaban mejoran notablemente durante el proceso, ahondando en precisión y calidad.

@tic revista d'innovació educativa ha estado comprometida siempre con el movimiento del Open Access y así lo manifestó en su documento fundacional.

Gracias a su política de acceso abierto y a su rápida inclusión en bases de datos, bibliotecas e índices como DOAJ, Dialnet, Scientific Commons, WorldCat, ROAR, OAIster, Dulcinea, Sherpa-Romeo, Recolecta o las bibliotecas que forman parte de REBIUN, la revista ganó en visibilidad, y al poco tiempo, fue evaluada y aceptada en Latindex, 
RESH (2010), MIAR (2011), DICE (2012), CARHUS Plus (2014), CIRC, ERIH Plus (2015), REDIB, Dialnet Métricas (2018), etc.

En el año 2016 @tic revista d'innovació educativa consiguió el Sello de Calidad de FECYT y también fue indexada en Emerging Sources Citation Index (ESCI) lo que supuso un hito para nuestra publicación.

Tradicionalmente la dirección de la revista ha recaído en la directora o director del Servei de Formació Permanent i Innovació Educativa. Así, desde su fundación en el año 2008 y hasta el número 12 (Primavera de 2014) la dirección estuvo en manos de la catedrática de Lingüística General de la Universitat de València, Beatriz GallardoPaúls. Desde el número 13 (Otoño de 2014) y hasta el número 20 (Primavera de 2018), este cargo recayó en la profesora de la Facultat de Filosofia i Ciències de l'Educació, María Jesús Martínez Usarralde, y en el último número, su director fue Ferran Suay i Lerma, profesor de la Facultat de Psicología de la Universitat de València. A partir del cambio de nombre la dirección ha pasado a ser compartida; así, el actual director del SFPIE, Ferran Suay i Lerma comparte la dirección de Research in Education and Learning Innovation Archives (REALIA) con el profesor Alejandro Armellini, de Northampton University.

\section{PIONERA Y ALGUNOS HITOS}

@tic revista d'innovació educativa fue la primera revista de la Universitat de València en emplear el sistema de publicación electrónica Open fournal System (OfS). Hacia 2008 el Servei d'Informàtica de la UV había instalado en sus servidores esta aplicación creada por el Public Knowledge Project, pero no fue hasta la puesta en marcha de la iniciativa de nuestra revista cuando este sistema se comenzó a usar. En julio de 2019 ya son más de 80 las revistas que emplean el OJS, sistema instalado en nuestros servidores universitarios. No todas las revistas de esta institución lo emplean y no son solo revistas de esta institución las que lo emplean; asociaciones vinculadas a esta se han sumado con el paso de los años.

Por otro lado, también estuvo entre las primeras que comenzaron a emplear el $\mathrm{Di}$ gital Object Identification (DOI) en sus artículos, gracias a la actuación del Servei de Biblioteques i Documentació (SBD), quien lo puso en marcha y lo coordina desde el año 2012. Este servicio facilitó su implementación retroactiva, así que desde su puesta en marcha y en escasas semanas todos nuestros artículos desde el número 1 contaban con un DOI identificativo universal.

\section{UN CAMBIO NECESARIO}

Desde 2009 un elemento que había causado confusión en el equipo editorial es la diversidad con la que se citaba la publicación. Era obvio que en la creación del nombre no se habían tenido en cuenta recomendaciones indispensables de consulta como la que Delgado López-Cózar, Ruiz-Pérez, y Jiménez-Contreras (2006) habían hecho sobre el título de una publicación; estos recomiendan que:

En la medida de lo posible debe rechazarse el uso de abreviaturas y acrónimos en el título. Aunque un determinado acrónimo pueda ser muy bien conocido en un ámbito profesional o especialidad muy concreta, fuera de esta puede provocar dudas y confusiones. En ningún caso se comenzará el título con el acrónimo o sigla. (2006, p. 111).

Si bien este extenso documento de Delgado López-Cózar et al. (2006) junto al de Giménez-Toledo et al. (2001) resultaron de tan gran utilidad al equipo de la revista para su adecuación y mejora, a esta recomendación llegamos tarde. 
Ello había dado lugar a que, por poner un ejemplo, en Scopus, se mencione la revista hasta de 12 formas diferentes, veamos:

- @tic. revista d’innovació educativa

- @Tic. Revista d'Innovacióeducativa

- @tic. Revistadïnnocacióeducative

- @Tic. Revista D’innovació Educativa

- @Tic Revista d’innovacio Educativa

- @tic. Revista d'innovació educativa

- En @tic Revista de Innovación Educativa

- @tic, Revista d'Innovació Educativa

- Revista dinnovació Educativa. Servei De Formació Permanent I Innovació Educativa

- Revista d'innovació educativa

- Revista D’innovació Educativa

- @Tic, Revista D’innovació Educativa

Por eso en 2018 se decidió que la revista asumiría nuevos retos. Entre ellos el más radical sería el cambio de nombre. El finalmente escogido ya sabe el lector que es: Research in Education and Learning Innovation Archives (REALIA), que el portal del ISSN ha incorporado vinculándolo a @tic revista d'innovació educativa de la siguiente forma (ver Figura 1).

No es este el primer ejemplo de revistas que a lo largo de la historia de la publicación científica española han llevado a cabo un cambio de nombre. En nuestra área, la de la educación, encontramos algunos ejemplos de importantes títulos que a lo largo de los años tomaron esta decisión por las razones más diversas (ver Tabla 2).

La Revista de Escuelas Normales que ya hemos mencionado y que fue fundada en 1923 por un equipo de profesores "de las Normales de Guadalajara" (Díez-Torre, Del Pozo-Andrés, y Segura-Redondo, 1988, p. 12) entre los que destacó Modesto Bargalló (su editor principal en sus primeros años), se transformó en 1987 en Revista Interuniversitaria de Formación del Profesorado.

La Revista galego-portuguesa de psicoloxía e educación: revista de estudios e investigación en psicología y educación fundada en 1997, simplificó su nombre por el de Revista de estudios e investigación en psicología y educación en el año 2014.

Otro ejemplo es el de la revista de la Universidad de Salamanca denominada Teoría de la Educación: Educación y Cultura en la Sociedad de la Información (TESI), que desde el 2014 continúa publicándose bajo el título Education in The Knowledge Society (EKS).

O la revista de la Universitat Oberta de Catalunya, conocida como RUSC. Revista de universidad y sociedad del conocimiento o por su nombre en inglés como RUSC. Universities and Knowledge Society fournal, que desde 2015 es publicada por el grupo Springer bajo el nombre de International fournal of Educational Technology in Higher Education (ETHE), manteniendo su histórico vínculo con la UOC y el más reciente de la Universidad de Los Andes (Colombia). Sin embargo, el listado es más extenso, como se puede ver en la Tabla 2. 


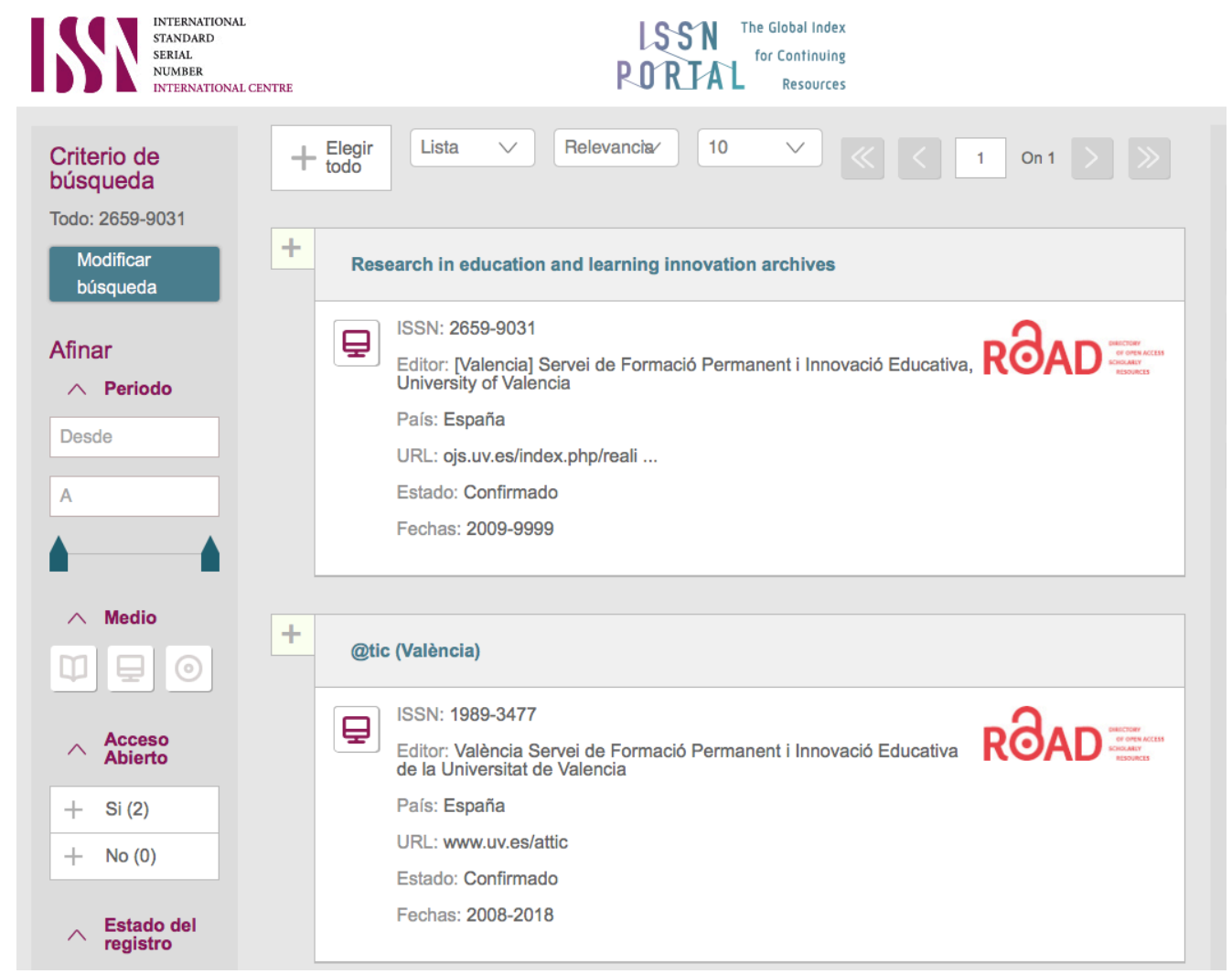

Figura 1. Registro de ambas revistas en el portal del ISSN

Tabla 2. Listado de algunas de las revistas científicas españolas que han cambiado su nombre en las últimas décadas

\begin{tabular}{|c|c|c|c|}
\hline ISSN & Título anterior & ISSN nuevo & Es continuada por... \\
\hline 0213-8638 & $\begin{array}{l}\text { Revista de Escuelas Normales (1923-1936) } \\
\text { (1970) }\end{array}$ & $\begin{array}{l}0213-8646 / \\
2530-3791\end{array}$ & $\begin{array}{l}\text { Revista interuniversitaria de formación } \\
\text { del profesorado (1987-) }\end{array}$ \\
\hline 0210-2846 & $\begin{array}{l}\text { Revista del Centro de Estudios Extremeños } \\
(1927-1944)\end{array}$ & 0210-2854 & Revista de estudios extremeños (1945-) \\
\hline 0034-8716 & Revista de Política Internacional (1950-1979) & 0210-9794 & $\begin{array}{l}\text { Revista de Estudios Internacionales } \\
(1980-1986)\end{array}$ \\
\hline 0034-8155 & $\begin{array}{l}\text { Revista de Estudios Agrosociales } \\
(1952-1994)\end{array}$ & $1575-1198$ & $\begin{array}{l}\text { Revista española de estudios } \\
\text { agrosociales y pesqueros (1998-) }\end{array}$ \\
\hline 0211-8394 & Agricultura y Sociedad (1976-1998) & $1575-1198$ & $\begin{array}{l}\text { Revista española de estudios } \\
\text { agrosociales y pesqueros (1998-) }\end{array}$ \\
\hline $0210-4873$ & $\begin{array}{l}\text { Papeles del Laboratorio de Arqueología de } \\
\text { Valencia (1962-1975) }\end{array}$ & 0210-3729 & $\begin{array}{l}\text { Saguntum. Papeles del Laboratorio de } \\
\text { Arqueología de Valencia (1977-) }\end{array}$ \\
\hline $0210-5241$ & $\begin{array}{l}\text { Papeles del Departamento de Geografía } \\
\text { (1968-1979) }\end{array}$ & $1989-4627$ & Papeles de geografía (1984-) \\
\hline 0210-0924 & $\begin{array}{l}\text { Revista de Instituciones Europeas } \\
(1974-1996)\end{array}$ & $1138-4026$ & $\begin{array}{l}\text { Revista de Derecho Comunitario } \\
\text { Europeo (1997-) }\end{array}$ \\
\hline 0211-3228 & $\begin{array}{l}\text { Cuadernos de prehistoria de la Universidad } \\
\text { de Granada (1976-1992) }\end{array}$ & $2174-8063$ & $\begin{array}{l}\text { Cuadernos de prehistoria y arqueología } \\
\text { de la Universidad de Granada (2008-) }\end{array}$ \\
\hline 0211-0849 & $\begin{array}{l}\text { Cuadernos de historia moderna y } \\
\text { contemporánea (1980-1987) }\end{array}$ & 0214-4018 & Cuadernos de historia moderna (1988-) \\
\hline 0211-0849 & $\begin{array}{l}\text { Cuadernos de historia moderna y } \\
\text { contemporánea (1980-1987) }\end{array}$ & $0214-400 x$ & $\begin{array}{l}\text { Cuadernos de historia contemporánea } \\
(1988-)\end{array}$ \\
\hline
\end{tabular}




\begin{tabular}{|c|c|c|c|}
\hline \multicolumn{4}{|c|}{ Table 2 continued } \\
\hline ISSN & Título anterior & ISSN nuevo & Es continuada por... \\
\hline $0211-6111$ & Quinto centenario (1981-1990) & $1132-8312$ & $\begin{array}{l}\text { Revista complutense de historia de } \\
\text { América (1991-) }\end{array}$ \\
\hline 0213-1390 & $\begin{array}{l}\text { Estudios humanísticos. Geografía, historia y } \\
\text { arte (1984-2001) }\end{array}$ & $1696-0319$ & $\begin{array}{l}\text { De arte revista de historia del arte } \\
(2002-)\end{array}$ \\
\hline 0213-1390 & $\begin{array}{l}\text { Estudios humanísticos. Geografía, historia y } \\
\text { arte (1984-2001) }\end{array}$ & $1696-0300$ & Estudios humanísticos. Historia (2002) \\
\hline $1133-0805$ & $\begin{array}{l}\text { Cuadernos de psiquiatría y psicoterapia } \\
\text { infantil (1985-1997) }\end{array}$ & $1575-5967$ & $\begin{array}{l}\text { Cuadernos de psiquiatría y psicoterapia } \\
\text { del niño y del adolescente (1998-) }\end{array}$ \\
\hline 0213-7321 & Stvdivm. Filología (1985-1993) & $1137-8417$ & $\begin{array}{l}\text { Studium Revista de humanidades } \\
\text { (1995-) }\end{array}$ \\
\hline 0213-8085 & $\begin{array}{l}\text { Studium. Geografía, historia, arte, filosofia } \\
(1987-1993)\end{array}$ & $1137-8417$ & $\begin{array}{l}\text { Studium Revista de humanidades } \\
\text { (1995-) }\end{array}$ \\
\hline 0213-358x & $\begin{array}{l}\text { Revista del Círculo Vasco del Arbitraje } \\
\text { (1986-1989) }\end{array}$ & $0214-7246$ & $\begin{array}{l}\text { Revista vasca de derecho procesal y } \\
\text { arbitraje = Zuzenbide prozesala ta } \\
\text { Arbitraia euskal aldizkaria (1989-) }\end{array}$ \\
\hline 0213-554X & $\begin{array}{l}\text { Revista vasca de derecho procesal } \\
(1987-1989)\end{array}$ & $0214-7246$ & $\begin{array}{l}\text { Revista vasca de derecho procesal y } \\
\text { arbitraje = Zuzenbide prozesala ta } \\
\text { Arbitraia euskal aldizkaria (1989-) }\end{array}$ \\
\hline $1133-1259$ & $\begin{array}{l}\text { BFD: Boletín de la Facultad de Derecho de la } \\
\text { UNED (1977-2006) }\end{array}$ & 2255-3436 & $\begin{array}{l}\text { Revista de Derecho UNED (RDUNED) } \\
(2006-)\end{array}$ \\
\hline $1886-7642$ & $\begin{array}{l}\text { V.O.: revista de cine en versión original } \\
(1994-2000)\end{array}$ & $1886-7227$ & Versión Original Revista de cine (2000-) \\
\hline $1138-1663$ & $\begin{array}{l}\text { Revista galego-portuguesa de psicoloxía e } \\
\text { educación : revista de estudios e } \\
\text { investigación en psicología y educación } \\
\text { (1997-2013) }\end{array}$ & $2386-7418$ & $\begin{array}{l}\text { Revista de estudios e investigación en } \\
\text { psicología y educación (2014-) }\end{array}$ \\
\hline $1137-5868$ & $\begin{array}{l}\text { Revista del Ministerio de Trabajo e } \\
\text { Inmigración* (1997-2011) }\end{array}$ & 2174-7504 & $\begin{array}{l}\text { Revista del Ministerio de Empleo y } \\
\text { Seguridad Social (2011-) }\end{array}$ \\
\hline $1138-9737$ & $\begin{array}{l}\text { Teoría de la Educación: Educación y Cultura } \\
\text { en la Sociedad de la Información (TESI) } \\
\text { (1999-2014) }\end{array}$ & $2444-8729$ & $\begin{array}{l}\text { Education in The Knowledge Society } \\
\text { (EKS) (2014-) }\end{array}$ \\
\hline $1578-4568$ & $\begin{array}{l}\text { Revista de estudios de la Administración } \\
\text { Local (REAL)* (2001-2003) }\end{array}$ & $1699-7476$ & $\begin{array}{l}\text { Revista de estudios de la } \\
\text { administración local y autonómica } \\
\text { (REALA) (2004-2011) }\end{array}$ \\
\hline 1698-580X & $\begin{array}{l}\text { Revista de universidad y sociedad del } \\
\text { conocimiento (2004-2015) }\end{array}$ & 2365-9440 & $\begin{array}{l}\text { International Journal of Educational } \\
\text { Technology in Higher Education (2016-) }\end{array}$ \\
\hline
\end{tabular}

* Las revistas con un asterisco han vivido más de un cambio de denominación.

Estamos seguros de que todas estas publicaciones se enfrentaron a las dificultades inmediatas que un cambio de nombre de publicación implica; esperamos poder superarlas cuanto antes.

Antes de formalizar este nuevo título, el equipo editorial sí tuvo en cuenta en esta ocasión los criterios de los expertos.

Por otra parte y como ya hemos apuntado, este cambio ha ido de la mano de algunos otros; por ejemplo:

- De forma lógica, la revista pasa a alojarse en un nuevo espacio del Open fournal System de la Universitat de València. Su nueva dirección es: https://www.uv.es/re alia o también: https://ojs.uv.es/index.php/realia/index

- La revista cuenta con un nuevo diseño y un logo que realza el acrónimo, si bien el nombre con el que la revista quiere que se le mencione es: Research in Education and Learning Innovation Archives 
- El equipo editorial ha incorporado un nuevo miembro en la dirección, el profesor Alejandro Armellini experto en tecnologías educativas y conocedor del mundo académico hispanohablante y anglófono, que se une a la fructífera experiencia académica y profesional del profesor Ferran Suay i Lerma

- Research in Education and Learning Innovation Archives ha decidido centrarse en un tipo concreto de artículos (1. Investigaciones, 2. Informes, estudios y propuestas, 3. Artículos de posición, 4. Revisiones y metaanálisis de la literatura concluyentes, todos ellos siempre que sean de interés para un público internacional y que realicen aportaciones significativas al campo de conocimiento), y limitar las contribuciones de experiencias educativas

- Todos los artículos incorporan una sección resumen inicial en la que el autor debe especificar: qué se conocía sobre el tema objeto del artículo, qué novedades aporta este artículo y las implicaciones que tiene para la práctica o la política

- Se ha añadido la identificación de autores mediante el sistema ORCID de forma generalizada

- Al formato PDF de publicación se incorporan dos nuevas versiones: la HTML (para la lectura en pantalla) y la epub (para la lectura en dispositivos móviles)

- La revista se adhiere a los principios éticos de publicación presentados por el Committee on Publication Ethics (COPE), Principles of Transparency and Best Practice in Scholarly Publishing Committee on Publication Ethics (COPE/DOAJ/OASPA/WAME, 2018)

- Al igual que hicimos con @tic revista d'innovació educativa, hemos abierto un canal de difusión de información en Twitter. Nuestro usuario es: @REALIA10174486

Estos cambios irán acompañados progresivamente de algunos más, derivados de la creciente profesionalización de la edición científica y los requerimientos técnicos de los diferentes planes de Ciencia Abierta de España y la UE. Entre otro está la puesta en marcha de las métricas alternativas, la integración con repositorios de preservación digital, el uso de XML-JATS, el depósito avanzado de metadatos y su accesibilidad, etc.

\section{CONCLUSIONES}

En este texto hemos explicado las razones que nos han llevado a cambiar el título de la revista que durante diez años el SFPIE ha editado por el de Research in Education and Learning Innovation Archives (REALIA).

Este cambio ha ido acompañado de algunos otros, más bien técnicos y formales, aunque también nos ha permitido definir con mayor claridad su perfil para adecuarlo a las exigencias de un entorno cada vez más especializado. La revista seguirá publicando originales en tres lenguas: español, inglés y valenciano aún consciente de la dificultad que la no primacía de el inglés entraña. Hemos apuntado cuáles son algunos de los retos que nos quedan por delante, y esperamos poder avanzar y consolidarnos con la ayuda de todos ustedes: lectores, autores y revisores.

\section{REFERENCIAS}

Aliaga, F. M. (2014). Veinte años de publicación electrónica y de acceso abierto: la madurez de una pionera. RELIEVE - Revista ELectronica de Investigacion y EValuacion Educativa, 20(1), 1-11. https://doi.org/10.7203/relieve.20.1.3856 
COPE/DOAJ/OASPA/WAME. (2018). Principles of Transparency and Best Practice in Scholarly Publishing Committee. Descargado de https://publicationethics.org/files/ Principles_of_Transparency_and_Best_Practice_in_Scholarly_Publishingv3.pdf

Delgado López-Cózar, E. (2015). Las revistas electrónicas en acceso abierto: pasado, presente y futuro. RELIEVE- Revista ELectrónica de Investigación y EValuación Educativa, 21(1), 1-15. https://doi.org/10.7203/relieve.21.1.5005

Delgado López-Cózar, E., Ruiz-Pérez, R., y Jiménez-Contreras, E. (2006). La edición de revistas científicas. Directrices, criterios y modelos de evaluación. Granada: Universidad de Granada. Descargado de https://www.fecyt.es/es/system/files/publications/attachments/2014/11/ la_edicion_de_revistas_cientificas._directrices_criterios_y_modelos_de_evaluacion.pdf

Díez-Torre, A., Del Pozo-Andrés, M. M., y Segura-Redondo, M. (1988). La "Revista de Escuelas Normales": publicación de regeneración normalista nacida en Guadalajara (1923-1936). Revista interuniversitaria de formación del profesorado, 1, 9-29. Descargado de https://dialnet.unirioja.es/descarga/articulo/117529.pdf

García-Hoz, V. (1980). La educación en la España del siglo XX. Revista española de pedagogía, 38(150), 99-120. Descargado de https://revistadepedagogia.org/wp-content/uploads/ 2018/04/7-La-Educacion-en-la-Espa $\{\{$ n\}\}a-del-Siglo-XX.pdf

Giménez-Toledo, E., Gómez-Caridad, I., Martín-Sempere, M.-J., Páez-Mañá, J., Román, A., Urdín, C., y Vázquez-Valero, M. (2001). La edición de revistas científicas: guía de buenos usos (A. Román Román, Ed.). Madrid: CSIC /CINDOC. Descargado de http://eprints.rclis.org/7764/1/GU\char"00CD \relaxA.pdf

Moreno-Pulido, A., López-González, M. A., Rubio-Garay, F., Saúl, L. A., y Sánchez-Elvira-Paniagua, A. (2013). Evolución de las revistas españolas de Ciencias Sociales en el Journal Citation Reports (2006-2010) y su valoración como indicio de calidad en la normativa evaluadora española. Revista Española de Documentación Científica, 36(3). https://doi.org/10.3989/redc.2013.3.987

OCE-UV. (s.f.). Innovació educativa, $1^{a}$ fase (2003-2006). Descargado June 1, 2019, de https://www.uv.es/ oce/1fasecontext.htm

Rodríguez-Yunta, L., y Giménez-Toledo, E. (2013). Fusión, coedición o reestructuración de revistas científicas en humanidades y ciencias sociales. El profesional de la información, 22(1), 36-45. Descargado de http://www.elprofesionaldelainformacion.com/contenidos/2013/enero/05.pdf

Ruiz-Corbella, M., Galán, A., y Diestro, A. (2014). Las revistas científicas de educación en España: evolución y perspectivas de futuro. RELIEVE - Revista ELectronica de Investigacion y EValuacion Educativa, 20(2), 1-27.

https://doi.org/10.7203/relieve.20.2.4361 